\title{
Fibrilação Atrial e Acidente Vascular Cerebral
}

\author{
Atrial Fibrillation and Stroke \\ Rubens José Gagliardi', Vivian Dias Baptista Gagliardi
}

\section{RESUMO}

Cerca de 20 a $30 \%$ dos acidentes vasculares cerebrais (AVC) são de origem cardioembólica, sendo a fibrilação atrial (FA) a principal causa cardiogênica. A FA é uma situação não rara na população e aumenta sua prevalência com a idade. Está relacionada a quadros de AVC mais graves, comparativamente às demais causas. A prevenção de AVC em pacientes com FA deve ser realizada com anticoagulação oral, sendo a varfarina a medicação mais utilizada; no entanto, esta medicação é de difícil controle, devido a múltiplas interaçôes medicamentosas, interaçóes alimentares, o que faz necessário manter monitorização laboratorial periódica. Metade dos pacientes que iniciam este tratamento o interrompe em meses e metade dos tratados não atinge o alvo terapêutico. Como evento adverso, existe o risco de hemorragias, incluindo hemorragia cerebral, e o benefício da medicação deve ser individualizado ao paciente. Nesta atualização são apresentadas considerações sobre a farmacologia dos anticoagulantes e resultados de recentes ensaios clínicos. Neste cenário, os novos anticoagulantes orais (como a dabigatrana, rivaroxabana, apixabana, edoxabana) têm surgido como opção à varfarina, por apresentarem-se com eficácia semelhante ou discretamente melhor do que a varfarina e com risco de hemorragia discretamente menor.

Unitermos. Acidente Vascular Cerebral, Fibrilação Atrial, Varfarina, Anticoagulantes.

Citação. Gagliardi RJ, Gagliardi VDB. Fibrilação Atrial e Acidente Vascular Cerebral.

\begin{abstract}
Approximately 20-30\% of strokes are cardioembolic, and atrial fibrillation (AF) is the main cardiogenic cause. $\mathrm{AF}$ is not an uncommon issue in the population, and its prevalence increases with age and is related to a more severe stroke. The prevention of stroke in patients with $\mathrm{AF}$ should be performed with oral anticoagulation, and warfarin is the most frequently used medication; however, this medication is difficult to be managed due to multiple drug interactions and dietary interactions, which demands a periodical laboratory monitoring. Half of the patients who started on this treatment interrupt it in months, and half of the treated patients do not reach the therapeutic target. In addition, there is a risk of bleeding, including cerebral hemorrhage, and the benefit of the medication should be individualized to each patient. In this scenario the new oral anticoagulants (such as dabigatran, rivaroxaban, apixaban, edoxaban) have emerged as an option to warfarin, which present with similar or slightly better efficacy than warfarin and risk of hemorrhage slightly lower.
\end{abstract}

Keywords. Stroke, Atrial Fibrillation, Warfarin, Anticoagulants.

Citation. Gagliardi RJ, Gagliardi VDB. Atrial Fibrillation and Stroke.

\footnotetext{
Trabalho realizado da Faculdade de Ciências Médicas da Santa Casa de Sáo Paulo, Sáo Paulo, São Paulo-SP, Brasil.

1.Neurologista, Professor Titular de Neurologia da Faculdade de Ciências Médicas da Santa Casa de Sáo Paulo, Chefe da Disciplina de Neurologia da Santa Casa de São Paulo, São Paulo-SP, Brasil.

2.Residente da Clínica Neurológica da Santa Casa de São Paulo, São Paulo-SP, Brasil.
}

Endereço para correspondência: Rubens José Gagliardi Disciplina de Neurologia da Santa Casa de São Paulo. Rua Dr. Cesário Motta Jr., 112 CEP 01221-020, São Paulo-SP, Brasil.

E-mail: rubensjg@apm.org.br 


\section{INTRODUÇÃO}

O Acidente Vascular Cerebral (AVC) ${ }^{1}$ é a principal causa de morte e de sequelas no Brasil ${ }^{2}$ sendo o cardioembolismo responsável por 20 a 30\% de todos os AVC 3 . Entre as causas cardiogênicas, a fibrilaçâo atrial (FA) é a principal ${ }^{4}$. Destaca-se ainda que o AVC cárdio-embólico costuma ser mais grave comparativamente ao AVC trombótico, com índice de mortalidade e desabilidade duas vezes maior do que as demais formas de AVC e com mortalidade de até $24 \%$ em 30 dias $^{5-7}$. O envolvimento de recursos econômicos no tratamento deste tipo de AVC também é mais elevado, comparativamente as demais causas $^{8}$. Vários fatores podem explicar este achado, como maior prevalência de comorbidades nestes pacientes e maior risco de complicações e interações com as medicações associadas 5 .

Existe uma relação muito forte entre AVC e FA. A FA aumenta em cinco a oito vezes o risco de AVC e poucas situaçóes na medicina são tão significativas, quanto à detecção de um fator de risco, a possibilidade da sua correção e os benefícios daí alcançados. A FA é um fator de risco extremamente forte para o AVC e deve ser devidamente abordada. Muitos doentes com diagnóstico de AVC criptogênico, quando são submetidos a uma investigação exaustiva, mostram que se trata de uma FA, ou eventualmente de outra arritmia cardíaca. Este fato destaca a importância de uma investigação etiológica completa, visando uma correta prevenção do AVC nestes doentes que podem beneficiar-se com a profilaxia da anticoagulação?.

A FA é uma situação não rara na população geral, estimando uma prevalência em torno de 1,5 a $2 \%$; aumenta significativamente com a idade, sendo $0,5 \%$ nos indivíduos de 40 anos e 10\% nos indivíduos acima de 80 anos de idade ${ }^{9-11}$. É interessante lembrar que um terço das FA se apresentam com características familiares ${ }^{4}$, merecendo maior atenção neste grupo de pessoas. Um terço dos pacientes com FA não tem sintomas e não recebem tratamento preventivo específico ${ }^{10}$.

$\mathrm{O}$ risco de AVC em doentes com FA é diretamente ligado à idade, sendo que entre 50 a 59 anos o risco é de $1,5 \%$ ao ano; pacientes entre 80 a 90 anos o risco se eleva para $23,5 \%$ ao $\mathrm{ano}^{4,12}$. Outras comorbidades, como hipertensão arterial, diabetes, insuficiência cardíaca, doença vascular periférica e AVC prévios, aumentam significativamente o risco do AVC. O tratamento preventivo com anticoagulação tem benefícios em todas as idades ${ }^{13}$.

A prevenção do AVC nos doentes com FA deve ser preferencialmente realizada com anticoagulação oral ${ }^{9,13-}$ 15. A varfarina é a medicação mais largamente utilizada, foi introduzida há mais de 50 anos e é um fármaco muito bem conhecido quanto aos seus benefícios e riscos. Vários estudos clínicos de grande porte, têm mostrado excelentes resultados com a varfarina, evidenciando uma redução do risco relativo do AVC, em média, de 60 a $70 \%$ e da mortalidade em $26 \%{ }^{13-15}$. Há uma redução de $2.7 \%$ ao ano de todos os tipos de AVC para a prevenção primária e $8.4 \%$ ao ano para a prevenção secundária, quando comparada a dose ajustada de varfarina ao placebo ou ausência de tratamento ${ }^{14}$. A anticoagulação sem dúvida é uma medida eficaz e segura para a prevenção do AVC frente a uma FA; tem indicação classificada como nível de evidência/recomendação $1 \mathrm{~A}$, em diferentes consensos ${ }^{9,16}$. Devido a estes resultados, a varfarina reinou por décadas como uma medicação de escolha nesta situação.

A varfarina atua como antagonista da vitamina $\mathrm{K}$, em vários fatores de coagulação. Bloqueia diferentes estágios da cascata de coagulação, reduzindo a síntese de vitamina $\mathrm{K}$ nos fatores vitamina $\mathrm{K}$ dependentes (fatores II, VII, IX e X). Tem características farmacocinéticas e farmacodinâmicas específicas que podem facilitar ou dificultar o seu manuseio e os resultados clínicos. As principais características são: administração oral, início de ação lento, absorção rápida e completa, ligação forte às proteínas plasmáticas (>98\%), somente o fármaco livre é farmacologicamente ativo. Possui uma vida média de 36-42 horas, baseada na ação em diferentes fatores de coagulação, com tempos diferentes nos diferentes fatores (fator II: 60 horas; fator VII: 6 horas; fator IX: 24 horas; fator X: 40 horas).

Outra característica desta droga que merece ser mencionada é a frequente e importante interação com alimentos (como por exemplo verduras), com álcool e com outras medicaçóes, situaçôes que comprometem a eficácia e aumentam o risco de complicaçóes hemorrágicas. O médico, ao prescrever este medicamento, deve obrigatoriamente orientar o paciente para abster-se de uma série de alimentos e bebidas com álcool ou manter a 
mesma composição alimentar em todas as refeições, e evitar a associação com determinados fármacos, para minimizar o risco de uma possível interação, que pode reduzir ou potencializar a açáo anticoagulante da varfarina.

Os pacientes em uso da varfarina devem ser monitorados laboratorialmente com dosagens sanguíneas do INR (International Normalized Ratio), que deve se manter entre valores de 2 a 3 , considerados como seguros e eficazes. Índices do INR abaixo de 2 não tem suficiente eficácia e superiores a 3, associa-se a maior risco de hemorragia. Este índice é bastante instável e deve ser repetido com frequência (semanal até o equilibrio da dose e em seguida quinzenalmente ou mensalmente).

Apesar da eficácia comprovada da varfarina algumas consideraçóes importantes devem ser feitas, como $^{17}$ :

- 60\% dos pacientes com indicação da varfarina, não estão medicados.

- Metade dos pacientes que iniciaram o tratamento, o interrompem em questão de meses.

- Entre os medicados, metade não está no alvo terapêutico.

Fornari et al. ${ }^{18}$ mostraram que, em média, dos pacientes que deveriam estar em uso de varfarina apenas $46,5 \%$ estão medicados e destes apenas $15,6 \%$ se encontram dentro do nível terapêutico desejado. Uma ampla metanálise ${ }^{19}$, envolvendo 124.551 pacientes mostrou que entre os doentes que estavam recebendo adequadamente a varfarina, apenas 54 a $62 \%$ se mantinham dentro da faixa terapêutica adequada - TTR ("time in therapeutic range"). Na nossa experiência, através de estudo realizado no setor de doenças cerebrovasculares da Disciplina de Neurologia da Santa Casa de São Paulo, observamos que, num período de três anos de acompanhamento, 55\% dos nossos pacientes em uso da varfarina se mantinham dentro do alvo terapêutico desejado (INR entre 2 e 3) 20 . Os principais motivos para a falta de efetividade com este tratamento foram: perda de motivação sobre os cuidados a serem seguidos e a importância do tratamento regular, falta de compreensão por parte do paciente com o tratamento, falta de orientação por parte do médico prescritor, dificuldade em retornar às frequentes consultas (para fazer os controles) e limitação econômica ${ }^{20}$. É importante o conhecimento destas situaçóes, para se tentar minimi- zar estes impasses frente à necessidade desta medicação.

A varfarina possui algumas vantagens para a sua prescrição, como: baixo custo, controle laboratorial eficaz e seguro, existência de antídoto para o caso sangramento ou risco de hemorragia e por ser uma droga bem conhecida. Possui, entretanto, algumas desvantagens, como: necessidade de frequente monitorização do INR, instabilidade plasmática, frequente interação com alimentos, drogas e álcool e estreita janela terapêutica.

Os pacientes com FA e que por qualquer motivo não possam ser medicados com anticoagulantes, podem para a prevenção do AVC, receber como alternativa, um antiagregante plaquetário (clopidogrel, AAS), porém os resultados são significativamente inferiores, como demonstrou o estudo ACTIVE W'21, sendo esta opção reservada apenas para situaçóes específicas e bem definidas (impossibilidade de emprego do anticoagulante) ${ }^{21,22}$.

O risco do emprego do anticoagulante é a hemorragia, principalmente a hemorragia cerebral, que pode ser fatal. Para avaliar o risco de sangramento em pacientes com FA em uso de varfarina, alguns estudos, como por exemplo o HAS-BLED (Hypertension, Abnormal renall liver function, Stroke - Bleeding history or predisposition, Labile international normalized ratio [INR], Elderly, Drugs/alcohol), HEMORR ${ }_{2} \mathrm{HAGES} \mathrm{(Hepatic} \mathrm{or} \mathrm{renal} \mathrm{disea-}$ se, Ethanol abuse, Malignancy, Older age, Reduced platelet count or function, Re-bleeding, Hypertension, Anemia, Genetic factors, Excessive fall risk, and Stroke) e o ATRIA (AnTicoagulation and Risk factors In $A F)^{23}$, tem sido conduzidos oferecendo ferramentas para análise e redução do risco de hemorragia. Todos estes três estudos oferecem orientações com pequena capacidade discriminatória para estratificação do risco de hemorragia. Estabelecer o risco de sangramento é um grande desafio e tem sido mais difícil do que estabelecer o risco de tromboembolismo no doente com FA.

As limitaçóes e dificuldades no manuseio da varfarina levaram a procura de outros fármacos com função anticoagulante, que se apresentassem com maior estabilidade, menor risco e eventual melhor resposta clínica. Assim, começou surgir, a partir de 2004 uma nova família de anticoagulantes, chamados de novos anticoagulantes orais (NACO). O ximelagatran foi o primeiro NACO disponível, porém foi retirado do uso clínico em 2006, 
devido a sua hepatotoxicidade. Desde entâo, dabigatrana, rivaroxabana, apixabana, edoxabana e outros, têm sido investigados em grandes ensaios clínicos randomizados e agora representam alternativas válidas para a varfarina na prevençâo de acidente vascular cerebral em doentes com FA não-valvar.

Os novos anticoagulantes orais recentemente adicionados ao arsenal terapêutico tem um mecanismo de ação diferente da varfarina, pois atuam inibindo a trombina (dabigatrana) ou o fator Xa (rivaroxabana, apixabana, edoxabana) e se apresentam como alternativas para os pacientes que necessitam de anticoagulação, para a prevenção de tromboembolismo em doentes com fibrilação atrial não valvular ${ }^{24}$. Provavelmente causarão impacto no tratamento destes doentes. Destas, a dabigatrana e a rivaroxabana estão aprovados para uso no Brasil, na prevenção do AVC e os demais, acredita-se que sejam aprovados em breve.

Os ensaios clínicos com estas medicações (estudos RE-LY' ${ }^{25}$, ROCKET AF ${ }^{26}$, ARISTOTLE ${ }^{27}$, AVERRO$\mathrm{ES}^{28}$, ENGAGE $\mathrm{AF}^{29}$ ) mostraram que estas drogas são seguras, com eficácia semelhante ou discretamente melhor do que a varfarina e com risco de hemorragia discretamente menor, variando com a dose empregada e o tipo de paciente. A dabigatrana tem importante metabolização renal e assim deve ser evitada em doentes com insuficiência renal; a rivaroxabana e a apixabana tem metabolização hepática devendo ser ponderado o seu uso nos doentes com insuficiência deste órgão.

Recentes metanálises, analisando estes grandes ensaios clínicos, considerando as limitações através de comparaçóes de estudos com diferentes desenhos, mostram que estas novas medicações tem eficácia semelhante a varfarina e menor risco de hemorragia, inclusive de hemorragia cerebral ${ }^{24,30,31}$.

Entre os novos anticoagulantes devemos lembrar as seguintes possíveis interações medicamentosas: dabigatrana com verapamil, quinidina, amiodarona, claritromicina, rifanpicina; rivaroxabana e apixabana com inibidores do citocroma P450 3A4 (CYP3A4), os antimicóticos azole, ritonavir e claritromicina.

O efeito máximo dos NACO na coagulação ocorre com aproximadamente 3 horas após a tomada da medicação; nesta fase temos o pico da concentração plasmática.
O seu efeito desaparece rapidamente, em 12 a 24 horas após a ingestão do medicamento ${ }^{25-28}$.

As principais restrições ao emprego dos novos anticoagulantes orais são: não existe um método laboratorial eficaz para medir a atividade anticoagulante, pouca experiência clínica, falta de antídoto específico, custo elevado.

Em conclusão, podemos citar que os novos anticoagualantes orais se apresentam como opção ao tratamento preventivo do AVC em doentes com FA e são candidatos aos novos anticoagulantes os pacientes que não conseguem controle adequado com a varfarina, não aderem ao tratamento regular, não realizam satisfatoriamente controles do INR e os pacientes com maior risco de sangramento. A varfarina não deve ser abandonada do nosso arsenal terapêutico.

\section{REFERÊNCIAS}

1.Gagliardi RJ. Acidente Vascular Cerebral ou Acidente Vascular Encefálico? Qual a melhor nomenclatura? Rev Neurocienc 2010;18:131-132.

2.Ministério da Saúde. Mortalidade-Brasil. Óbitos por Ocorrência por Sexo Segundo Causa CID-BR-10. Available at: http//:www.datasus.gov.br/Estatísticas Vitais - Mortalidade e Nascidos Vivos/Mortalidade Geral/Abrangência Geográfica: Brasil por regiāo e Unidade de Federação. Acessado 01.05.2010 3.Wolf PA, Abbott RD, Kannel WB. Atrial fibrillation as an independent risk factor for stroke: the Framingham Study. Stroke 1991;22:983-988.

http://dx.doi.org/10.1161/01.STR.22.8.983

4.Marini C, De Santis F, Sacco S, Russo T, Olivieri L, Totaro R, et al. Contribution of atrial fibrillation to incidence and outcome of ischemic stroke: results from a population-based study. Stroke 2005;36:1115-1119.

http://dx.doi.org/10.1161/01.STR.0000166053.83476.4a

5.McGrath, ER, Fang J, O'Conghaile A, O'Donnell M. Association of atrila fibrillation with mortality and disability after schemic stroke. Neurology 2013;81:825-832.

http://dx.doi.org/10.1212/WNL.0b013e3182a2cc15

6.Lin HJ, Wolf PA, Kelly-Hayes M, Beiser AS, Kase CS, Benjamin EJ, et al. Stroke severity in atrial fibrillation. The Framingham Study. Stroke 1996;27:1760-1764.

http://dx.doi.org/10.1161/01.STR.27.10.1760

7.Dulli DA, Stanko H, Levine RL. Atrial fibrillation is associated with severe acute ischemic stroke. Neuroepidemiology 2003;22:118-123.

http://dx.doi.org/10.1159/000068743

8.Bruggenjurgen B, Rossnagel K, Roll S, Andersson FL, Selim D, Müller-Nordhorn J, et al. The impact of atrial fibrillation on the cost of stroke: the Berlin acute stroke study. Value Health 2007;10:137-143.

http://dx.doi.org/10.1111/j.1524-4733.2006.00160.x

9.Fuster V, Ryden LE, Cannom DS, Crijns HJ, Curtis AB, Ellenbogen KA, et al. ACC/AHA/ESC 2006 guidelines for the management of patients with atrial fibrillation. A report of the American College of Cardiology/American Heart Association Task Force on Practice Guidelines and the European Society 
of Cardiology Committee for Practice. J Am Coll Cardiol 2006; 48: 854-906. http://dx.doi.org/10.1016/j.jacc.2006.07.009

10.European Heart Rhythm Association, European Association For Cardio-Thoracic Surgery, Camm AJ, Kirchhof P, Lip GY, et al. Guidelines for the management of atrial fibrillation: the task force for the management of atrial fibrillation of the European Society of Cardiology (Esc). Eur Heart J 2010;31:2369-429.

http://dx.doi.org/10.1093/eurheartj/ehq278

11.Loewen P, Dahri K. Risk of bleeding with oral anticoagulants: an updated systematic review and performance analysis of clinical prediction rules, Ann Hematol 2011;90:1191-1200.

http://dx.doi.org/10.1007/s00277-011-1267-3

12.Jørgensen HS, Nakayama H, Reith J, Raaschou HO, Olsen TS. Acute stroke with atrial fibrillation: the Copenhagen Stroke Study. Stroke 1996;10:1765-69. http://dx.doi.org/10.1161/01.STR.27.10.1765

13.You JJ, Singer DE, Howard PA, et al. American College of Chest Physicians. Antithrombotic therapy for atrial fibrillation: Antithrombotic Therapy and Prevention of Thrombosis, 9th ed: American College of Chest Physicians Evidence-Based Clinical Practice Guidelines. Chest 2012;141(2 Suppl.):e531S-e575S.

14.Atrial Fibrillation Investigators. Risk factors for stroke and efficacy of antithrombotic therapy in atrial fibrillation. Analysis of pooled data from five randomized controlled trials. Arch Intern Med 1994;154:1449-1457.

http://dx.doi.org/10.1001/archinte.1994.00420130036007

http://dx.doi.org/10.1001/archinte.154.13.1449

15.Hart RG, Pearce LA, Aguilar MI. Meta-analysis: antithrombotic therapy to prevent stroke in patients who have nonvalvular atrial fibrillation. Ann Intern Med 2007;146:857-867.

http://dx.doi.org/10.7326/0003-4819-146-12-200706190-00007

16.Camm AJ, Kirchhof P, Lip GYH, Ulrich Schotten, Schotten U, Savelieva I, Ernst S, et al. Guidelines for the management of atrial fibrillation. The Task Force for the Management of Atrial Fibrillation of the European Society of Cardiology (ESC). Eur. Heart J 2010;31:2369-2429.

http://dx.doi.org/10.1093/eurheartj/ehq278

17.Camm J. Atrial fibrillation. Amer College Cardio. Scientif Sessions 2012.

18.Fornari LS, Calderaro D, Nasser IB et al. Misuse of antithrombotic therapy in atrial fibrillation patients: frequent, pervasive and persistent. J Thromb Thrombolysis 2007;23:65-71.

http://dx.doi.org/10.1007/s11239-006-9012-9

19.Rose AJ, Hylec E, Ozonoff A, Ash AS, Reisman JI, Berlowitz AB, et al. Risk-ajusted percent in therapeutic range as a quality indicator for outpatient oral anticoagulation. Cir Cardiovasc Qual outcomes 2011;4:22-29.

http://dx.doi.org/10.1161/CIRCOUTCOMES.110.957738

20.Gagliardi VDB \& Gagliardi RJ. Fatores que interagem na estabilidade da anticoagulação com varfarina em doentes com acidente vascular cerebral. Cong
Paulista Neurologia, Anais, 2013.

21.The ACTIVE Writing Group on behalf of the ACTIVE Investigators. Clopidogrel plus aspirin versus oral anticoagulation for atrial fibrillation in the Atrial fibrillation Clopidogrel Trial with Irbesartan for prevention of Vascular Events (ACTIVE W): a randomised controlled trial. Lancet 2006;367:1903-1912. http://dx.doi.org/10.1016/S0140-6736(06)68845-4

22.ACTIVE Investigators, Connolly SJ, Pogue J, Hart RG, et al. Effect of clopidogrel added to aspirin in patients with atrial fibrillation. N Engl J Med 2009;360:2066-2078.

http://dx.doi.org/10.1056/NEJMoa0901301

23.Apostolakis S, Lane DA, Guo Y, Buller H, Lip GYH. Performance of the HEMORR2HAGES, ATRIA, and HAS-BLED Bleeding Risk-Prediction Scores in Patients With Atrial Fibrillation Undergoing Anticoagulation. J Am Coll Cardiol 2012.

http://dx.doi.org/10.1016/j.jacc.2012.06.019

24.Lip GY, Larsen TB, Skjoth F, Rasmussen LH. Indirect comparisons of new anticoagulant drug for efficacy and safety when used for stroke prevention in atrial fibrillation. J Am Coll Cardiol 2012:60:738-746.

http://dx.doi.org/10.1016/j.jacc.2012.03.019

25.Connolly SJ, Ezekowitz MD, Yusuf S, Eikelboom J, Oldgren J, Parekh A, et al. RE-LY Steering Committee and Investigators. Dabigatran versus warfarin in patients with atrial fibrillation. N Engl J Med 2009;361:1139-1151.

http://dx.doi.org/10.1056/NEJMoa0905561

26.Patel MR, Mahaffey KW, Garg J, Pan G, Singer DE, Hacke W, et al. ROCKET AF Investigators. Rivaroxaban versus warfarin in nonvalvular atrial fibrillation. N Engl J Med 2011;365:883-891.

http://dx.doi.org/10.1056/NEJMoa1009638

27.Granger CB, Alexander JH, McMurray JJ, Lopes RD, Hylek EM, Hanna $\mathrm{M}$, et al. ARISTOTLE Committees and Investigators. Apixaban versus warfarin in patients with atrial fibrillation. N Engl J Med 2011;365:981-992.

http://dx.doi.org/10.1056/NEJMoa1 107039

28.Connolly SJ, Eikelboom J, Joyner C, Diener HC, Hart R, Golitsyn S, et al. AVERROES Steering Committee and Investigators. Apixaban in patients with atrial fibrillation. N Engl J Med 2011;364:806-817.

http://dx.doi.org/10.1056/NEJMoa1007432

29.Giugliano RP, Ruff CT, Braunwald E, Murphy SA, Wiviott SD, Halperin $\mathrm{JL}$, et al. Edoxaban versus warfarin in patients with atrial fibrillation. $\mathrm{N}$ Engl J Med 2013;369:2093-2104.

http://dx.doi.org/10.1056/NEJMoa1310907

30.Ruff CT, Giugliano RP, Brauwald E, et al. Comparison of the efficacy and safety of new oral anticoagulants with warfarin in patients with atrial fibrillation: a meta-analysis of randomised trilas. Lancet 2013.

31. Chatterjee S, Sardar P, Biondi-Zoccai G, Kumbhani DJ. New oral anticoagulants and the risk of intracranial hemorrhage. JAMA 2013.

http://dx.doi.org/10.1001/jamaneurol.2013.4021 